\section{(1)}

CrossMark

\title{
In vivo growth of 60 non-screening detected lung cancers: a computed tomography study
}

\author{
Onno M. Mets ${ }^{1}$, Kaman Chung ${ }^{2}$, Pieter Zanen ${ }^{3}$, Ernst T. Scholten ${ }^{2}$, \\ Wouter B. Veldhuis ${ }^{1}$, Bram van Ginneken ${ }^{2}$, Mathias Prokop ${ }^{4}$, \\ Cornelia M. Schaefer-Prokop ${ }^{2,5}$ and Pim A. de Jong ${ }^{1}$
}

Affiliations: ${ }^{1}$ Dept of Radiology, University Medical Center Utrecht, Utrecht University, Utrecht, The Netherlands. ${ }^{2}$ Diagnostic Image Analysis Group, Radboud University Medical Center, Nijmegen, The Netherlands. ${ }^{3}$ Dept of Pulmonology, University Medical Center Utrecht, Utrecht University, Utrecht, The Netherlands. ${ }^{4}$ Dept of Radiology, Radboud University Medical Center, Nijmegen, The Netherlands. ${ }^{5}$ Dept of Radiology, Meander Medical Center, Amersfoort, The Netherlands.

Correspondence: Onno M Mets, Dept of Radiology, University Medical Center Utrecht, Heidelberglaan 100 , 3584 CX Utrecht, the Netherlands. E-mail: o.m.metsdumcutrecht.nl

@ERSpublications

Lung cancers in vivo conform to an exponential growth pattern http://ow.ly/ps0L30jN1LD

Cite this article as: Mets $\mathrm{OM}$, Chung $\mathrm{K}$, Zanen $\mathrm{P}$, et al. In vivo growth of 60 non-screening detected lung cancers: a computed tomography study. Eur Respir J 2018; 51: 1702183 [https://doi.org/10.1183/ 13993003.02183-2017].

ABSTRACT Current pulmonary nodule management guidelines are based on nodule volume doubling time, which assumes exponential growth behaviour. However, this is a theory that has never been validated in vivo in the routine-care target population. This study evaluates growth patterns of untreated solid and subsolid lung cancers of various histologies in a non-screening setting.

Growth behaviour of pathology-proven lung cancers from two academic centres that were imaged at least three times before diagnosis $(n=60)$ was analysed using dedicated software. Random-intercept random-slope mixed-models analysis was applied to test which growth pattern most accurately described lung cancer growth. Individual growth curves were plotted per pathology subgroup and nodule type.

We confirmed that growth in both subsolid and solid lung cancers is best explained by an exponential model. However, subsolid lesions generally progress slower than solid ones. Baseline lesion volume was not related to growth, indicating that smaller lesions do not grow slower compared to larger ones.

By showing that lung cancer conforms to exponential growth we provide the first experimental basis in the routine-care setting for the assumption made in volume doubling time analysis. 


\section{Introduction}

Scientific literature contains few articles dealing with in vivo growth of human lung cancers. This has several probable reasons, including the most obvious one: cancers undergo immediate treatment after diagnosis. Furthermore, sophisticated volumetric software became available only recently, and an increasing number of small cancers are found incidentally due to improved computed tomography (CT) technology and increasing numbers of CT examinations.

An exponential growth pattern is one of the most prominent patterns in cell biology and was suggested for malignancies 60 years ago [1]. The key concept of exponential growth is that the growth rate (i.e. the number of cells added over time) increases as the tumour gets larger. This is due to the fact that each tumour cell divides into two cells, starting from one into two, next into four, then eight, 16, and so on. While exponential growth seems intuitive, prior studies into tumour growth gave contradictory results, ranging from confirmation of exponential growth [2] to varying growth patterns, including Gompertzian (i.e. reaching a plateau) or irregular patterns over time [3]. A more recent animal study suggested exponential growth in lung cancers in mice [4]. Only two CT studies have evaluated growth patterns of pulmonary malignancies in humans $[5,6]$. These studies both used lung cancer screening data, and reached opposing conclusions.

The issue of in vivo natural behaviour of lung cancer has become increasingly important for medical decision making, as we are dealing with an ever increasing number of incidentally found pulmonary nodules. Nodule management is frequently dealt with using current guideline algorithms [7-9], which are based mainly on nodule volume doubling time (VDT). The formula assumes exponential growth behaviour. However, this has never been tested in the routine-care target population of the guidelines. Therefore, this study evaluates the in vivo behaviour of untreated solid and subsolid lung cancers of various histology in a non-screening setting.

\section{Methods}

This study is part of a larger Dutch multicentre project to evaluate pulmonary nodules in non-screening CT imaging. The need to obtain informed consent was waived by the ethical review board of both the University Medical Center Utrecht and the Radboud University Medical Center Nijmegen (14-384/C and 2014/172, respectively), due to the retrospective study design.

\section{Subjects}

We retrospectively collected data from all subjects aged $>40$ years who underwent CT of the chest between 2004 and 2012 in two university centres ( $n=16850$ and $n=23454)$. No selection based on imaging indication or in-/outpatient status was made. We linked all these subjects to the Dutch National Cancer Registry to assess who developed lung cancer in the interval between CT imaging and the end of 2014. This yielded 3219 lung cancer cases. Lung cancer location and date of diagnosis were available from the National Cancer Registry. Those with an interval $<2$ months between the first available CT and the date of lung cancer diagnosis were excluded, as we aimed to investigate the longitudinal evolution of malignant pulmonary nodules without treatment. We excluded CT scans when mechanical ventilation, substantial consolidation or collapse or severe motion/breathing artefacts were present. In addition, lesions that could not be segmented properly due to a central location, extensive cavitation or chest wall invasion were excluded. Lastly, only cases with at least three separate CT scans were included, to allow growth pattern analysis of the lung cancer. Due to the retrospective study design smoking history and risk factors were not available. Figure 1 presents the flow chart of the study population.

\section{Nodule annotation}

All chest CT scans were assessed visually and annotated by one of two observers; a fourth-year radiology resident with a $\mathrm{PhD}$ in chest imaging and 7 years of experience in thoracic radiology, and a senior radiologist with $>30$ years of experience in chest radiology. Volume of lung cancers was semi-automatically assessed using a dedicated nodule software workstation (CIRRUS Lung Screening; Diagnostic Image Analysis Group, Nijmegen, the Netherlands and Fraunhofer MEVIS, Bremen, Germany). This software enables image viewing in different window/level settings, provides reconstructions in all three orthogonal planes and allows adaptation of the nodule borders.

\section{CT scanning}

Since imaging was performed for various clinical indications, different scan protocols were applied. Scans were obtained at different scanners of the same vendor in one centre (Philips Healthcare), and at various scanners of four different vendors in the other centre. The majority of scans (63\%) were thin-slice examinations (i.e. $\leqslant 1 \mathrm{~mm}$ ), and nearly all $(96 \%)$ had image slices $\leqslant 3 \mathrm{~mm}$. Median (interquartile range (IQR); range) follow-up interval between the CT scans was 9.4 months (4.4-21.1 months; 1-72 months). 


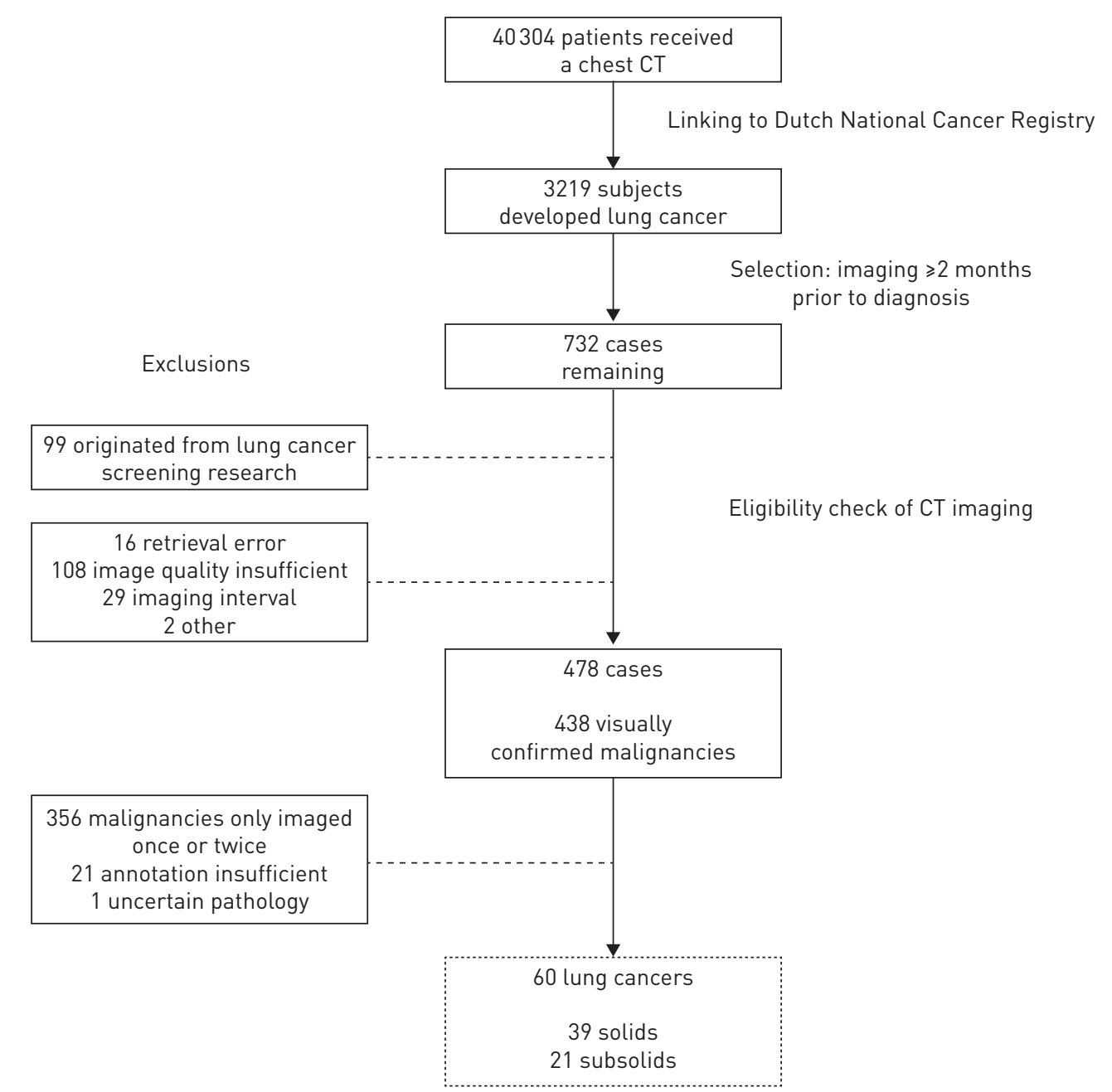

FIGURE 1 Flow chart of study population selection. CT: computed tomography.

\section{Statistical analysis}

A range of growth pattern models were fitted to the longitudinal data including linear, exponential and quadratic growth curves, as well as all the inversed relations. In this scenario, regression techniques are not the appropriate statistical method to compare the fit of various models, because data points of a single lesion are not independent, but show (rather strong) correlation. Therefore, random-intercept random-slope mixed-models analysis was applied. Which of the models most accurately described the measured growth over time was assessed by comparing the Akaike information criterion (AIC); the smaller the value, the better the fit. To assess the impact of baseline volume and nodule type on growth, we included both as covariates together with their interactions with time. Additionally, we plotted individual growth curves to illustrate the deviation of the observed nodule growth from the exponential model on a per lesion basis. Deviation was expressed as difference between virtual and real lesion volume, as percentage of total volume. Virtual lesion volume for follow-up data points was calculated assuming an exponential increase between the first and last data point.

Comparison between groups was performed using Mann-Whitney U-testing (continuous data) or Chi-squared testing (proportions). All statistical analyses were performed using SPSS 22 (SPSS, Chicago, IL, USA). A p-value $<0.05$ was considered significant.

\section{Results}

Study population

From the 478 lung cancer cases included in the complete nodule study project database, 60 pathologically proven lung cancers (in 55 different subjects) were included in this ancillary study. These were all imaged 
on at least three different time points. Characteristics are shown in table 1. Comparing the 55 included subjects with all remaining 423 subjects, we found no significant differences regarding sex or age ( $\mathrm{p}=0.38$ and 0.56 , respectively). However, survival at end of follow-up was significantly better $(\mathrm{p}=0.01)$ and histology of malignancies more often represented adenocarcinomas $(p<0.01)$. This was similar when compared to the subgroup that only received one or two CT examinations. When exponential growth was assumed and plotted between the two available data points in lung cancers imaged only twice, comparison showed that the growth rate for the included 60 lung cancers with three or more imaging time points was not significantly lower $(\mathrm{p}=0.08)$.

\section{Lung cancer growth analysis}

Individual growth patterns are illustrated in figure 2. In our clinical cohort of 60 lung cancers we found that an exponential model best fitted the data, illustrated by the fact that AIC values of the exponential model were by far the lowest (AIC 362 versus $>4400$ for the other models).

An exponential model is theoretically described by $\ln (y)=a+b x$, in which $a$ represents the intercept with the $\mathrm{y}$-axis and $b$ represents the growth rate. We derived the following equations in our dataset:

$$
\begin{gathered}
\text { Ln }(\text { volume subsolids })=8.09+0.58 \times \text { time in years } \\
\operatorname{Ln}(\text { volume solids })=6.69+1.09 \times \text { time in years }
\end{gathered}
$$

The higher intercept value for subsolids ( 8.09 versus $6.69, \mathrm{p}<0.01)$ indicates a difference in baseline lesion size between subsolid and solid lesions. The interaction between time and nodule type was significant, indicating a significantly different growth rate. Solid cancers grew faster than subsolid ones, reflected by the higher growth rate $(1.09$ versus $0.58, \mathrm{p}=0.02)$. The interaction between baseline volume and time was not significant, indicating that lesion volume does not determine growth rate.

Obviously, growth behaviour did not always exactly align with an ideal exponential curve. We found that in both solid and subsolid lung cancers, independent of histological subgroup, some over- and undershoot was present. Deviation between calculated and real lesion volume during follow-up showed a median (IQR; range) of $2 \%(-13-18 \% ;-42-112 \%$. Figure 3 visualises deviation from the ideal exponential growth line on a per-lesion basis.

\section{TABLE 1 Study population characteristics}

\begin{tabular}{lc} 
Sex & \\
Male & $29(53)$ \\
Female & $26(47)$ \\
Age years & $63(43-78)$ \\
Source & $26(47)$ \\
Centre 1 & 29 (53) \\
Centre 2 & 39 (65) \\
Nodule type & 21 (35) \\
Solid & 14 (5.5-41) \\
Subsolid & 1444 (90-36070) \\
Baseline diameter mm & \\
Baseline volume mm ${ }^{3}$ & 39 (65) \\
Pathology & 5 (8) \\
Adenocarcinoma & 8 (13) \\
Squamous cell carcinoma & 8 (13) \\
SCLC/NE & 17 (28) \\
Other & 15 (25) \\
Location & 5 (8) \\
RUL & $13(22)$ \\
LUL & 10 (17) \\
RML & \\
RLL & \\
LLL & \\
\hline Data are presented as n (\%) or median (range). The study population comprised 60 lung malignancies in 55 \\
subjects. SCLC: small cell lung carcinoma; NE: neuroendocrine; RUL: right upper lobe; LUL: left upper \\
lobe; RML: right middle lobe; RLL: right lower lobe; LLL: left lower lobe. \\
\hline
\end{tabular}




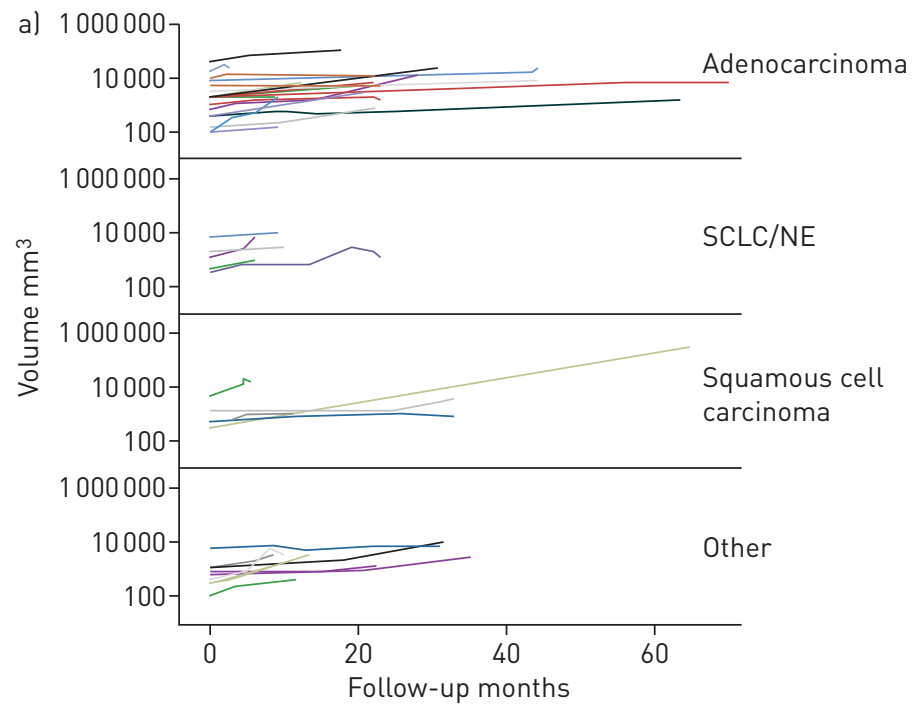

b)

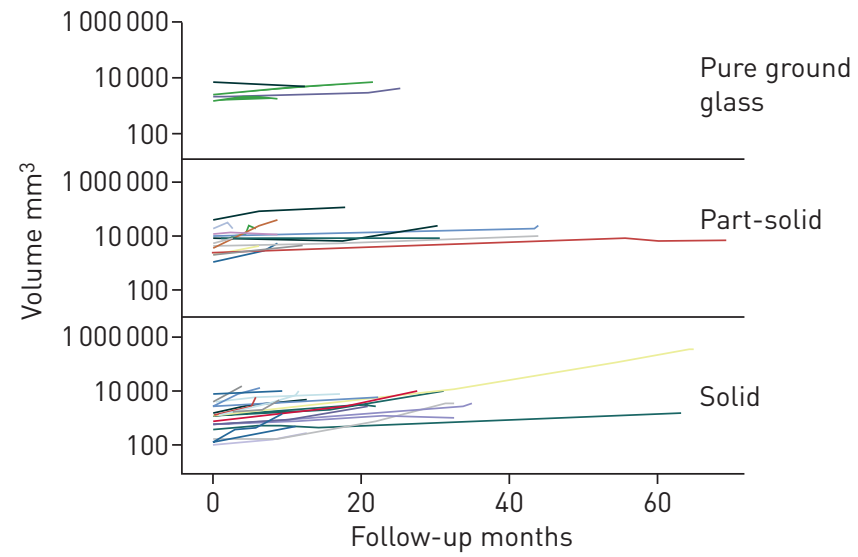

FIGURE 2 Individual growth patterns of 60 lung cancers per pathology subgroup and nodule subtype. Individual growth curves are given per a) pathology subgroup and b) nodule subtype. The volume (y-axis) is presented on a logarithmic scale; a perfectly exponential volume increase would show a straight line. Although in individual cases some deviation from a perfect straight line can be seen, mixed models analysis showed that this was insufficient to reject exponential growth as best fit to describe in vivo lung cancer behaviour in humans. SCLC: small cell lung carcinoma; NE: neuroendocrine.

\section{Discussion}

To our knowledge, this is the first study to evaluate in vivo growth of lung cancer in humans originating from a non-screening, daily routine-care setting. Our results from a substantially large cohort of lung cancers provide an experimental basis for the theoretically assumed exponential growth pattern.

Due to the fact that sequential CT imaging of untreated malignancies is rarely possible in humans, data on growth of human lung cancer are extremely limited in literature. The trend of more frequent CT imaging, improved CT technique and advanced software tools enabled us to study the growth behaviour of 60 histologically proven non-screen-detected malignancies. Since current management guidelines for the follow-up of incidentally and screen-detected nodules assume exponential growth to differentiate malignancies from benign lesions [7, 8], the validity of the growth behaviour model has become very important. Deviation of actual growth from the theoretical model may lead to under- or overtreatment of nodules.

Only two prior studies specifically analysed growth patterns of human lung cancers, both using lung cancer screening CT data [5, 6]. LINDELl et al. [5] reported a variety of growth curves in 18 medium-sized lung cancers (median $10.3 \mathrm{~mm}$ ), both solid and subsolid lesions. In their study one observer manually measured lesions diameter using callipers on fairly thick slices. Lesion volume was calculated assuming a perfect spherical lesion shape. Ultimately, plotted growth curves with at least four time points over a follow-up interval of at median 2.9 years were assessed visually. The authors concluded that in their cohort lung cancers were not limited to exponential growth and warned for misinterpretation using exponential-based equations [5]. In contrast, Heuvelmans et al. [6] assessed growth patterns more meticulously in 47 small solid screen-detected lung cancers with a median baseline volume of $100 \mathrm{~mm}^{3}$. They assessed growth curves with at least three time points over a median follow-up interval of a 2.1 years, using semi-automated nodule volumetry and quantitative analysis. They concluded that small- to intermediate-sized lung malignancies can generally be described by an exponential function [6]. Additionally, a small study by Quint et al. [11] assessed doubling times of various lung lesions, including 13 primary lung cancers. They showed that growth curves were visually fairly consistent with exponential increase; however, no further statistical analysis was performed. Differences in study populations with respect to number and type of nodules, as well as differences in growth measurement methods, are likely to have contributed to the opposing conclusions of the available studies [12].

In our study we used semi-automated volumetry instead of either manual measurements or visual growth curve assessment to analyse lung cancer growth over time, and included a fairly large number of 60 lung cancers. We included both solid and subsolid lesions, originating from a heterogeneous routine-care clinical population. Although data on risk factors and smoking history were unavailable in our study, our 

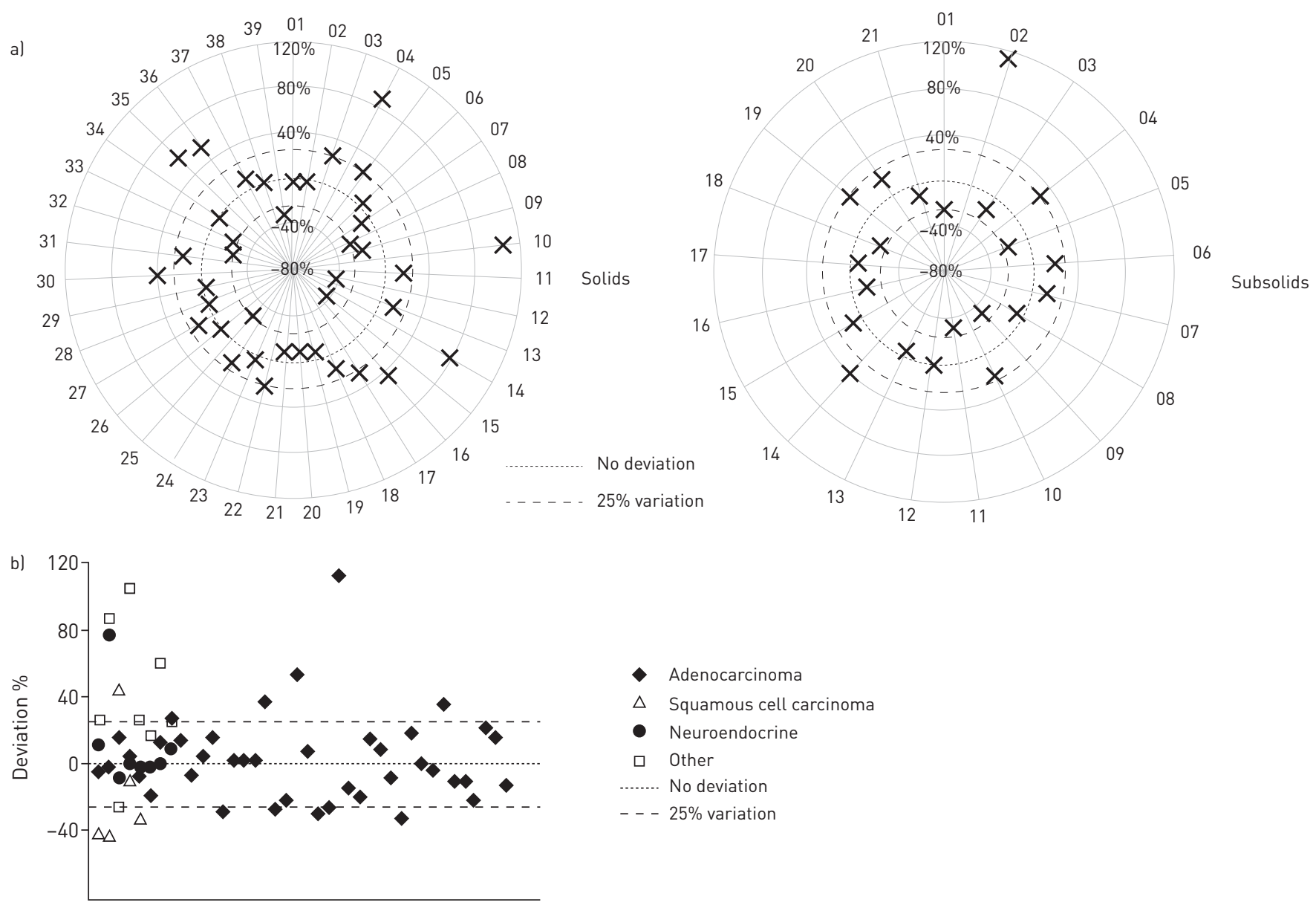

$$
\begin{aligned}
& \text { - Adenocarcinoma } \\
& \triangle \text { Squamous cell carcinoma } \\
& \text { - Neuroendocrine } \\
& \text { Other } \\
& \text {....... No deviation } \\
& \text { - - - } 25 \% \text { variation }
\end{aligned}
$$

FIGURE 3 Maximum deviation from ideal exponential growth on a per-lesion basis per nodule type and per pathology subgroup. Deviation was calculated by plotting a virtual exponential growth curve between the first and last data point of each individual lung cancer. This curve yielded virtual lesion volume for the imaging time points in between. Real lesion volume on these time points was then compared to the virtual lesion volume, and deviation was expressed as a percentage of the total volume. For each follow-up data point this deviation could theoretically either be zero (on the curve), positive (above the curve) or negative (below the curve). Maximum deviation was assessed for each lung cancer individually. a) In a lung cancer that perfectly follows exponential volume increase, there would be no difference between the real and virtual lesion volume, leading to a marker right on the dotted line in the spider web plots. As is seen, some deviation is present in both solid and subsolid lung cancers. The $25 \%$ volume difference is a recommended cut-off for measurement variations [8, 10]. b) Additionally, the scatter plot shows maximum deviation for individual lung cancers per pathology subgroup.

study population is essentially different from a homogeneous lung cancer screening population because it is not highly selected on age and smoking history. Additionally, all had a clinical indication for chest imaging, and prior malignancies were not excluded as they were in previous screening studies.

We confirmed statistically that growth in lung cancers was exponential in both solid and subsolid lesions, although on a per lesion base some deviation from the predicted exponential volume increase was seen. The magnitude of deviations showed a median close to zero (i.e. $2 \%$, IQR $-11-18 \%$ ), which falls inside the range of $25 \%$ volume change that has been posed as a threshold for significant volume change $[8,10]$. Variations in individual lung cancers will at least partly be due to technical differences between scans and some measurement errors, the latter being larger in smaller lesions and shorter follow-up [8, 10, 13]. Nevertheless, real deviations from the exponential growth may exist in some cases. However, these individual outliers were not sufficient to reject the conclusion that an exponential function best describes in vivo lung cancer growth in humans. Therefore, current clinical decision tools are correct in their assumption of exponential growth.

In addition to confirming the exponential nature of lung cancer growth in our dataset, we found that subsolid lesions are generally larger than solid ones at baseline, but show significantly slower progression over time. This is in line with previous literature that reported the generally smaller growth rate of subsolid lesions. As a consequence, management guidelines recommend larger intervals and a longer follow-up time for subsolid than for solid nodules [7, 8]. Although different in terms of baseline size and growth 
rate, both solid and subsolid nodules showed an exponential growth pattern. According to our results, baseline volume is not related to speed of growth over time, indicating that smaller lung lesions do not necessarily grow more slowly. Knowledge of individual growth behaviour may be used to further personalise follow-up intervals, which might vary for lesions within the same nodule type subgroup. This strategy is currently not effected in the guidelines, as intervals recommended are similar for all nodules within a certain subgroup. Future studies are needed to evaluate whether it is feasible, safe and cost effective to base follow-up strategy on individual growth behaviour instead of baseline volume only. However, it has to be noted that a total of three or more scans are needed to determine exponential behaviour. Therefore, it is most likely best applied in subsolid lesions that are often subject to long-term follow-up and in which overdiagnosis lingers [14], but it might also be applicable for a selected subgroup of slow-growing solid lesions.

The inherent and major limitation of our study is the possible selection bias towards smaller and more slowly growing lesions. Exponential growth pattern analysis requires at least three imaging time points, which automatically eliminates the fast-growing nodules and larger lesions that are more likely to be referred for immediate (therapeutic) actions without further follow-up. Growth rate differences between the included cancers with three or more imaging time points and lung cancers only imaged twice did not reach significance. Nevertheless, the possibility remains that some larger fast-growing lung cancers do not grow exponentially. Additionally, selection bias may have played a role in the difference we observed in baseline volume between solid and subsolid lesions. When smaller subsolid nodules have not progressed enough to warrant intervention/definitive diagnosis, they are not included. In addition, smaller subsolid nodules may be ignored and are more difficult to detect due to their morphology, which may be another reason they are not included in our study.

A second limitation is the retrospective nature of our study, which is less optimal due to the fact that the data are more heterogeneous. However, we believe a prospective design is ethically unacceptable in humans. Nevertheless, with this retrospective study design we were able to confirm the theory of the exponential growth model and echo the most recent data obtained in lung cancer screening setting.

In conclusion, we present the first study outside the lung cancer screening setting evaluating in vivo growth behaviour of solid and subsolid lung cancers in humans. We show that primary lung cancer growth conforms to an exponential model in both subsolid and solid lesions. This provides the first experimental basis in routine-care setting for the assumption made in VDT analysis. Therefore, current nodule management guidelines are correct in their assumption, and reasonable to use in the follow-up of lung nodules in routine care.

Acknowledgements: The authors thank the registration team of the Netherlands Comprehensive Cancer Organisation (IKNL) for the collection of data for the Netherlands Cancer Registry, as well as IKNL staff for scientific advice.

Support statement: This work was supported by the Dutch Cancer Foundation (grant number KUN-2013-6110). Funding information for this article has been deposited with the Crossref Funder Registry.

Conflict of interest: B. van Ginneken reports that he is a founder and shareholder of Thirona, and received grants and royalties from MEVIS Medical Solutions. M. Prokop reports receiving speaker's fees from Bracco, Bayer, Toshiba and Siemens. He received research funding from Toshiba.

\section{References}

1 Collins VP, Loeffler RK, Tivey H. Observations on growth rates of human tumors. Am J Roentgenol Radium Ther Nucl Med 1956; 76: 988-1000

2 Friberg S, Mattson S. On the growth rates of human malignant tumors: implications for medical decision making. J Surg Oncol 1997; 65: 284-297.

3 Retsky MW, Swartzendruber DE, Wardwell RH, et al. Is Gompertzian or exponential kinetics a valid description of individual human cancer growth? Med Hypotheses 1990; 33: 95-106.

4 Li M, Jirapatnakul A, Biancardi A, et al. Growth pattern analysis of murine lung neoplasms by advanced semi-automated quantification of micro-CT images. PLoS One 2013; 8: e83806.

5 Lindell RM, Hartman TE, Swensen SJ, et al. 5-year lung cancer screening experience: growth curves of 18 lung cancers compared to histologic type, CT attenuation, stage, survival, and size. Chest 2009; 136: 1586-1595.

6 Heuvelmans MA, Vliegenthart R, de Koning HJ, et al. Quantification of growth patterns of screen-detected lung cancers: the NELSON study. Lung Cancer 2017; 108: 48-54.

7 MacMahon H, Naidich DP, Goo JM, et al. Guidelines for management of incidental pulmonary nodules detected on CT images: from the Fleischner Society 2017. Radiology 2017; 284: 228-243.

8 Callister ME, Baldwin DR, Akram AR, et al. British Thoracic Society guidelines for the investigation and management of pulmonary nodules. Thorax 2015; 70: Suppl. 2, ii1-ii54.

9 American College of Radiology. Lung CT Screening Reporting and Data System (Lung-RADS). www.acr.org/ Quality-Safety/Resources/LungRADS Date last accessed: March 2015.

10 Devaraj A, van Ginneken B, Nair A, et al. Use of volumetry for lung nodule management: theory and practice. Radiology 2017; 284: 630-644. 
11 Quint LE, Cheng J, Schipper M, et al. Lung lesion doubling times: values and variability based on method of volume determination. Clin Radiol 2008; 63: 41-48.

12 Truong MT, Ko JP, Rossi SE, et al. Update in the evaluation of the solitary pulmonary nodule. Radiographics 2014; 34: 1658-1679.

13 Liang M, Yip R, Tang W, et al. Variation in screening CT-detected nodule volumetry as a function of size. AJR Am J Roentgenol 2017; 209: 304-308.

14 Hutchinson BD, Moreira AL, Ko JP. Spectrum of subsolid pulmonary nodules and overdiagnosis. Semin Roentgenol 2017; 52: 143-155. 